\title{
Buchbesprechungen · Book Reviews
}

Transfusionsmedizin Grundlagen -Therapie- Methodik Herausgeber: C. Mueller-Eckhardt Springer, Berlin, 1988 XV, 694 S., 97 Abb., gebunden DM 198,-ISBN 3-540-17059-6

Im deutschsprachigen Raum fehlte bislang eine detaillierte Zusammenstellung sowie eine Anleitung zum praktischen Vorgehen des klinisch tätigen Kollegen. Der konzeptionelle Aufbau des Buches mit umfassender Darstellung der transfusionsmedizinischen Historie bis hin zur Erläuterung der aktuellen rechtlichen Grundlagen des transfundierenden Arztes unterstreicht den Charakter eines Lehrbuches. Die wissenschaftliche Darlegung der Einzel-kapitel wird jeweils ergänzt durch eine umfangreiche Literaturzusammenstellung.

Auch wenn einzelne Kapitel, wie z.B. «Therapie mit Thrombozyten» sowie «Komplikationen und Nebenwirkungen von Bluttransfusionen» und «Physiologie der Blutgerinnung und Fibrinolyse», als besonders gelungene Abhandlungen hervortreten, wird dadurch nicht der Wert anderer Kapitel geschmälert, zumal sich jeder der Autoren in dem von ihm abgehandelten Kapitel durch eigene wissenschaftliche Arbeiten auszeichnet.

Als Nachteil des Bandes ist zu erwähnen, daß sich die einzelnen Aussagen bzw. Angaben in den verschiedenen Kapiteln z.T. untereinander widersprechen, so z.B. bzgl. der Indikationsstellung zur Thrombozytentransfusion bei Massiv-transfusion oder der zu fordernden Qualitätskriterien für Frischplasmen. Darüber hinaus ware bei der Vielzahl verwen-deter Abkürzungen ein entsprechendes Abkürzungs-verzeichnis wünschenswert, sowie ein Glossar am Ende des Buches mit Definitionen und Erläuterungen wichtiger Termine.

Dennoch steht mit dem Band «Transfusionsmedizin» ein Standardwerk im deutschsprachigen Raum zur Verfügung, das international anerkannten und etablierten Büchern gleichrangig ist. Die Schnelligkeit des wissenschaftlichen Erkenntniszuwachses auf dem Gebiet der Transfusionsmedizin macht allerdings eine regelmäßige Überarbeitung erforderlich, was besonders hier wünschenswert ware. Das Buch ist als Nachschlagewerk und Lehrbuch gleichermaßen für den klinisch als auch wissenschaftlich tätigen Arzt geeignet.

D. Söhngen, Düsseldorf

MSD-Manual der Diagnostik und Therapie

Urban \& Schwarzenberg, München

3060 S., 158 Abb., Kunststoffeinband, DM 98,-

ISBN 3-541-01854-2

Die deutsche Neuausgabe basiert auf der 15. englischsprachi-gen Auflage. Gegenüber der 3. deutschen Auflage von 1984 wurde das MSD-Manual in alien Teilen komplett überarbeitet und neu verfaßt. Mehr als 300 renommierte Wissenschaftler und Hochschullehrer garantieren für höchste Qualität und aktuellstes Wissen.

In 24 identisch nach Ätiologie, Pathogenese, Diagnostik, Therapie und Prophylaxe aufgebauten Kapiteln beschreibt das MSD-Manual 1724 Krankheitsbilder aus alien medizinischen Gebieten von der Augen- bis zur Zahnheilkunde. Ein eingestanztes Griffregister gestattet dem Leser einen umkom-plizierten, schnellen Zugriff auf das gesuchte Fachgebiet und die gezielte Information 
durch eine übersichtliche Gliederung auch innerhalb der einzelnen Abschnitte. So erlaubt das MSD-Manual Ärzten aller Fachrichtungen, sich in Notfallsituationen und Zweifelsfällen innerhalb des eigenen oder eines fremden Gebietes schnell und problemlos Sicher-heit und Klarheit über Symptomatik, Diagnose und Therapie zu verschaffen. Auf diese Weise ist das MSD gerade in einer Zeit der fortgeschrittenen Spezialisierung ein unersetzlicher Begleiter in der praktischen Arbeit jedes Arztes geworden.

P. T., München

Pocket Guide to Injectable Drugs

Herausgeber: L. A. Trissel

ASHP Publ. Records Dept., Berthesda, MD, 20814,1989

\$15.-

Das Handbook on Injectable Drugs von Lawrence A. Trissel gilt als Standardwerk für die Vermittlung von Stabilitäts- und Kompatibilitätsdaten parenteraler Arzneiformen. Die American Society of Hospital Pharmacists bietet dieses Nachschlagewerk nun auch als Taschenbuch an. Die darin enthaltenen Monographien der einzelnen Injektabilia geben Auskunft über den Arzneistoff, die Applikationsformen, die Dosierung, die Verdünnungsvorschrift, die Infusionsgeschwindigkeit und über die Stabilität. Die anschließenden Kompatibilitätstabel-len ermöglichen einerseits die Auswahl einer geeigneten Trägerlösung und vermitteln anderseits Inkompatibilitätsdaten mit gleichzeitig parenteral verabreichten Arzneimitteln. Das Buch liefert schnell eine Vielzahl von Informationen und ist daher für die Arbeit des Arztes und des Krankenhausapothe-kers auf der Station eine wertvolle Hilfe.

K. Schmidt, Schwäbisch Hall 